\title{
Asteroseismic measurement of surface-to-core rotation in a main-sequence star $^{\star}$
}

\author{
Donald W. Kurtz ${ }^{1, \text { a }}$, Hideyuki Saio ${ }^{2}$, Masao Takata ${ }^{3}$, Hiromoto Shibahashi ${ }^{3}$, Simon J. Murphy ${ }^{4}$, and \\ Takashi Sekii ${ }^{5}$
}

1 Jeremiah Horrocks Institute of Astrophysics, University of Central Lancashire, Preston PR1 2HE, UK

2 Astronomical Institute, Graduate School of Science, Tohoku University, Sendai, Miyagi 980-8578, Japan

3 Department of Astronomy, School of Science, The University of Tokyo, Bunkyo-ku, Tokyo 1130033, Japan

4 Sydney Institute for Astronomy, School of Physics, The University of Sydney, NSW 2006, Australia

5 National Astronomical Observatory of Japan, 2-21-1 Osawa, Mitaka, Tokyo 181-8588, Japan

\begin{abstract}
We have discovered rotationally split core g-mode triplets and surface pmode triplets and quintuplets in a terminal age main-sequence A star, KIC 11145123, that shows both $\delta$ Sct p-mode pulsations and $\gamma$ Dor g-mode pulsations. This gives the first robust determination of the rotation of the deep core and surface of a main-sequence star, essentially model-independently. We find its rotation to be nearly uniform with a period near $100 \mathrm{~d}$, but we show with high confidence that the surface rotates slightly faster than the core. A strong angular momentum transfer mechanism must be operating to produce the nearly rigid rotation, and a mechanism other than viscosity must be operating to produce a more rapidly rotating surface than core. Our asteroseismic result, along with previous asteroseismic constraints on internal rotation in some B stars, and measurements of internal rotation in some subgiant, giant and white dwarf stars, has made angular momentum transport in stars throughout their lifetimes an observational science.
\end{abstract}

\section{Introduction}

For four hundred years, since Galileo, we have known that the Sun rotates. As the Sun and stars evolve, their cores shrink while their outer envelopes expand and are eventually ejected, carrying away angular momentum. If stars conserved angular momentum throughout their lives, the surviving compact cores - white dwarfs and neutron stars - would spin much faster than is observed. Recently, Kepler Mission data have revealed core rotation in two red giant stars that is 5-10 times faster than the surface rotation [1] [2], a contrast weaker than expected. Therefore, a strong mechanism for angular momentum transport must be acting before stars become red giants, probably in the dominant mainsequence phase. Yet little is known of the internal rotation and angular momentum transport of stars.

Studies of stellar rotation and angular momentum transport are important for a full understanding of stellar evolution [3] [4]. This affects wider studies of the chemical evolution of the universe, of galaxy formation and evolution, through its strong impact on stellar structure and evolution. It is also closely connected to the stellar dynamo process, and hence contributes to our understanding of the origin of the magnetic fields in the universe.

$\star$ This proceedings paper is a digest of a paper by the same authors published in MNRAS, 444, 102 (2014); reprinted with permission

a e-mail: kurtzdw@gmail.com

This is an Open Access article distributed under the terms of the Creative Commons Attribution License 4.0, which permits unrestricted use, distribution, and reproduction in any medium, provided the original work is properly cited. 
In asteroseismology of main-sequence stars, pressure modes probe the outer layers of a star, and gravity modes probe the deep interior [5] [6]. To find both kinds of modes in one star promises a full view of the interior, a place Arthur Eddington [7] described as "less accessible to scientific investigation than any other region of our universe." Since the birth of helioseismology [8], observations of internal pulsational gravity modes have been eagerly sought, without clear success [9]. Consequently, for our Sun the interior differential rotation is known only half way down to the core [10]. Up to now the only other observational indications of internal rotation in main-sequence stars come from modeldependent studies of two $\beta$ Cep stars for which a single low overtone $\mathrm{p}$-mode rotational dipole triplet and two or three of five possible components of the lowest overtone g-mode quadrupole quintuplet have been observed. For the $\beta$ Cep star HD 129929 Dupret et al. [11] found indications of internal differential rotation (see also [12]), and for another $\beta$ Cep star, $\theta$ Oph, Briquet et al. [13] found indications of solid-body rotation. Both of these studies place only weak, model-dependent constraints on internal rotation.

Our aim is to find main-sequence stars that show both the pressure modes (p modes) and gravity, or buoyancy, modes (g modes) using the exquisitely precise Kepler Mission photometric data for the purpose of observing their interior rotation from the surface right to the core. The best candidates for this are stars known as $\delta$ Sct $-\gamma$ Dor hybrids, of which there are several hundred amongst the 190000 stars observed by Kepler during its four-year mission [14]. We discuss our first success, KIC 11145123, here.

\section{Observations and frequency analysis}

KIC 11145123 has a Kepler magnitude $\mathrm{Kp}=13$, and is a late A star. From the Kepler Input Catalogue (KIC) revised photometry [15], its effective temperature is $8050 \pm 200 \mathrm{~K}$ and its surface gravity is $\log g=4.0 \pm 0.2$ (cgs units), showing it to be a main-sequence A star. The data used for the analysis in this paper are the Kepler quarters 0 to $16(\mathrm{Q} 0-\mathrm{Q} 16)$ long cadence (LC) data. We used the multiscale, maximum a posteriori (msMAP) pipeline data; information on the reduction pipeline can be found in the data release notes $21^{1}$. To optimise the search for exoplanet transit signals, the msMAP data pipeline removes astrophysical signals with frequencies less than $0.1 \mathrm{~d}^{-1}$ (or periods greater than $10 \mathrm{~d}$ ). None of the pulsation frequencies we analyse in this paper are near to that lower limit, but if the star has a direct rotational signal, e.g. from starspots, that will have been erased by the pipeline. Since, as we show, the rotation period is near to $100 \mathrm{~d}$, any data reduction technique will struggle to find a direct signal at this period because of its similarity to the time span or the Kepler quarterly rolls. This has no effect on our analysis.

\section{Nearly model-independent inferences}

We measured the frequency splittings for the $15 \mathrm{~g}$-mode dipole triplets, and for a selection of p-mode triplets and quintuplets. For all of the p-mode multiplets, the splittings within the multiplet are equal within the formal errors. This shows that there are no second-order rotation effects measureable and that there is no strong magnetic field in the star, as that would perturb the multiplet frequency spacings. The equally split quintuplets indicate no detectable differential rotation in the latitudinal direction.

For high-overtone g modes the Ledoux constant asymptotically approaches $C_{n, l} \approx 1 / l(l+1)$, which for the $l=1$ dipole g modes in KIC 11145123 gives $C_{n, l} \approx 0.5$. This is a very general conclusion, with which our best models are certainly consistent. We find $P_{\text {rot }}$ (core) $=104-105 \mathrm{~d}$. On the other hand, using a typical value of the Ledoux constant for the p modes of our models, $C_{n, l}=0.03$, the p-mode triplets give surface rotation periods in the range $95-114 \mathrm{~d}$. Although the above estimates do not take account of errors in $C_{n, l}$, the consistent period range of the p modes with that of the g modes supports uniform rotation of the star in a broad way.

We can even show based on a careful argument about the average rotation rate, $\bar{\Omega}$, that the star is rotating differentially in the sense that the envelope rotation rate is slightly higher than the core rate.

\footnotetext{
${ }^{1}$ https://archive.stsci.edu/kepler/data_release.html
} 
We stress that the above result is based on only conservative assumptions that are not influenced by detailed modelling of the star and precise mode identification. For example, one of our fundamental assumptions is that the p mode is more sensitive to outer layers of the star than the g mode. This is generally true for any pair of a p mode and a high-order g mode in any main-sequence star. Moreover, although we rely on the identification of the g mode as the one with $l=1$ and a large radial order $(|n| \gg 1)$, the exact value of $n$ need not be specified, as is the case for the p mode. Therefore, our conclusion of the higher rotation rate in the envelope than in the core is robust.

\section{Discussion and conclusions}

These results are remarkable for several reasons. The most important of these is that we see surfaceto-core rotation clearly for the first time in a star burning hydrogen in the core. Secondly, the star is nearly a rigid rotator, but the surface layer for the highest amplitude p mode rotates more quickly than does the core. This is unexpected. Thirdly, the 100-d rotation period of KIC 11145123 is abnormally long for any nonmagnetic A star.

Amongst the magnetic Ap stars rotation periods of years are known; the longest is over a century for $\gamma$ Equ. However, we consider it unlikely that KIC 11145123 is currently a magnetic Ap star: the equal splitting of the p-mode multiplets argues against a magnetic field. No magnetic Ap star is known to pulsate in low-overtone p modes, or in g modes. While there is a class of rapidly oscillating Ap stars [16] that pulsate in high-overtone p modes, KIC 11145123 does not show any of these, and Saio [17] has shown that the strong magnetic fields of the Ap stars suppress the low-overtone p modes typical of $\delta$ Sct stars. All of these reasons argue against a magnetic field in KIC 11145123.

The high helium abundances of our best models suggest that KIC 11145123 could be an SX Phe variable that was formed in a binary system after significant mass accretion. If this is the case, then the envelope may have been spun up considerably. In this scenario, at present, near the end of mainsequence evolution, only a slight excess of angular frequency in the envelope remains and the rotation is nearly uniform and very slow. The small difference in the rotation rate between the core and the envelope suggests that the angular momentum transfer may be much stronger than has been previously thought. This is common to the requirement suggested from the recent discoveries that the rotation speeds of red giant cores, which are much slower than the theoretical predictions. High-resolution spectra will test this idea by determining abundances of, in particular, CNO, and will test our prediction above that KIC 11145123 is not a magnetic Ap star.

While the 100-d rotation period for KIC 11145123 is long for an A star, we note that Aerts et al. (2003) [12] found a similarly long 80-d rotation period in the early B star HD 129929 for which they found evidence of internal differential rotation. In the case of KIC 11145123 it is the slow rotation that makes the rotational splitting patterns so obvious. There are many hybrid $\delta$ Sct $-\gamma$ Dor stars in the Kepler data that show an abundance of g modes and p modes. But with the typically much faster rotation rate than for KIC 11145123, it is probable that the rotationally split multiplets for these stars are not equally split, because of second order effects. Then with the richness of the g-mode frequency spectrum, it is difficult to determine which of the plethora of frequency peaks in the amplitude spectrum belong to dipole or quadrupole multiplets in these faster rotators, since the multiplet patterns get mixed in frequency space. Progress may be made by understanding the slower rotators such as KIC 11145123 first, allowing us then to begin to understand the frequency patterns of more and more rapidly rotating stars. Studies of these stars benefit from the long 4-yr time-span of the Kepler data, since the mode frequency separations for the g modes are small.

Asteroseismology has revealed internal differential rotation in the outer half of the Sun [10], has put constraints on interior rotation of some main-sequence B stars [12] [13] [18] [19], surface-to-core differential rotation in two red giants stars [1] [2], and some subgiants [20] and both rigid rotation [21] and differential rotation [22] in two white dwarf stars.

Stars spend 90 per cent of their lifetimes as main-sequence stars. Now, for the first time, we have measured the rotation of a main-sequence A star, KIC 11145123, at the surface and in the core, essentially model-independently, and with a clarity never seen before. We have found it to be nearly a rigid rotator with the surface rotating slightly faster than the core. With this discovery, rotation and angular 
momentum transfer inside of main-sequence stars is now an observational science. Our understanding of KIC 11145123 also shows the direction for finding more main-sequence stars with similarly rich frequency spectra with many rotational multiplets for both $\mathrm{p}$ modes and g modes.

Stars are born in the wholly convective phase (the Hayashi phase), where uniform rotation is established because of large turbulent viscosity. Subsequent stellar evolution is generally a process of increasing the central mass concentration. A simple argument based on conservation of the local angular momentum then leads to the conclusion of a greater rotation rate in the central regions than in the envelope as a result of evolution. Following this scenario, we have modelled the evolution of angular frequency, $\Omega\left(M_{r}, t\right)$, for a hypothetical star similar to our best model for KIC 11145123 . We started with a uniformly rotating, fully convective protostar in its Hayashi phase and evolved it to the TAMS. In this model angular momentum is conserved in radiative layers, and in convective zones; initial uniform rotation was assumed and the total angular momentum was conserved. As the mainsequence evolution proceeds, a steep variation in $\Omega$ appears around the core. From the rotation kernels we conclude that we would have found differential rotation from core to surface in KIC11145123 of a factor of 5, had the star been born with uniform rotation and had angular momentum been conserved. Clearly, a strong mechanism for angular momentum transport must be acting to result in the nearly rigid rotation that we observe.

That the envelope of KIC 11145123 rotates more rapidly than the core puts a constraint on physical mechanisms of angular momentum transport. We may classify the transport mechanisms into two categories. In the first, the mechanisms operate only to reduce the gradient of the rotation rate. Viscosity (of any kind) is a representative example. On the other hand, the mechanisms in the second category can even reverse the sign of the gradient. Examples are the angular momentum transport by waves and mass accretion (including capture of planets and/or comets). Given the spin-up tendency of the core as a result of evolution, the mechanisms in the first category are clearly not sufficient to explain the more rapidly rotating envelope. We thus conclude that those in the second category must exist.

\section{References}

1. Deheuvels, S., García, R. A., Chaplin, W. J., et al., ApJ, 756, (2012) 19

2. Beck, P. G., Montalban, J., Kallinger, T., et al., Nature, 481, (2012) 55

3. Pinsonneault, M., ARA\&A, 35, (1997) 557

4. Tayar, J., \& Pinsonneault, M. H., ApJL, 775, (2013) LL1

5. Unno, W., Osaki, Y., Ando, H., Saio, H., \& Shibahashi, H., Nonradial oscillations of stars, Tokyo: University of Tokyo Press, (1989), 2nd ed.

6. Aerts, C., Christensen-Dalsgaard, J., \& Kurtz, D. W., Asteroseismology, Astronomy and Astrophysics Library. ISBN 978-1-4020-5178-4. Springer Science+Business Media B.V., (2010)

7. Eddington, A. S., The Internal Constitution of the Stars, Cambridge: Cambridge University Press, (1926) ISBN 9780521337083

8. Leighton, R. B., Noyes, R. W., \& Simon, G. W., ApJ, 135, (1962) 474

9. Appourchaux, T., Belkacem, K., Broomhall, A.-M., et al., A\&ARv, 18, (2010) 197

10. Schou, J., Antia, H. M., Basu, S., et al., ApJ, 505, (1998) 390

11. Dupret, M.-A., Thoul, A., Scuflaire, R., et al., A\&A, 415, (2004) 251

12. Aerts, C., Thoul, A., Daszyńska, J., et al., Science, 300, (2003) 1926

13. Briquet, M., Morel, T., Thoul, A., et al., MNRAS, 381, (2007) 1482

14. Uytterhoeven, K., Moya, A., Grigahcène, A., et al., A\&A, 534, (2011) AA125

15. Huber, D., Silva Aguirre, V., Matthews, J. M., et al., ApJS, 211, (2014) 2

16. Kurtz, D. W., ARA\&A, 28, (1990) 607

17. Saio, H., MNRAS, 360, (2005) 1022

18. Pamyatnykh, A. A., Handler, G., \& Dziembowski, W. A., MNRAS, 350, (2004) 1022

19. Dziembowski, W. A., \& Pamyatnykh, A. A., MNRAS, 385, (2008) 2061

20. Deheuvels, S., Doğan, G., Goupil, M. J., et al., A\&A, 564, (2014) AA27

21. Charpinet, S., Fontaine, G., \& Brassard, P., Nature, 461, (2009) 501

22. Córsico, A. H., Althaus, L. G., Kawaler, S. D., et al., MNRAS, 418, (2011) 2519 